\title{
Agrometeorological Indices, Crop Phenology and Yield of Pigeon pea as Influenced by Pigeon pea (Cajanus cajan L.) based Intercropping System
}

\author{
S. N. Chatte*, M. G. Jadhav, D. S. Dhekane, I. A. B. Mirza, \\ K. K. Dakhore and S. S. More \\ Department of Agriculture Meteorology, College of Agricultre, Vasantrao Naik Marathawada \\ Krishi Vidyapeeth, Parbhani 431 402, India \\ *Corresponding author
}

\section{Keywords}

Agrometeorologicla indices,

Intercropping,

Pigeon pea,

Phenophases,

Treatment

Article Info

Received:

14 October 2021

Accepted:

02 November 2021

Available Online:

10 November 2021

\section{A B S T R A C T}

A field investigation was conducted at experimental farm, Department of Agricultural Meteorology, located at college of Agriculture, V.N.M.K.V, Parbhani during kharif season of 2019-20. The experiment was laid out in RBD with three replication, under this study there were nine treatments viz. $\mathrm{T}_{1}$ (Pigeon pea + Sorghum), $\mathrm{T}_{2}$ (Pigeon pea + Maize), $\mathrm{T}_{3}$ (Pigeon pea + Soybean $), \mathrm{T}_{4}($ Pigeon pea + Sesamum), $\mathrm{T}_{5}$ (Pigeon pea), $\mathrm{T}_{6}$ (Sorghum), $\mathrm{T}_{7}$ (Maize), $\mathrm{T}_{8}$ (Soybean), $\mathrm{T}_{9}$ (Sesamum). In pigeon pea the highest total agrometeorological indices (GDD, HTU and PTU) accumulated by intercropped treatment $\mathrm{T}_{1}$ as compared to sole, by sorghum, maize and sesamum was highest in intercropped treatment i.e. $\left(T_{1}\right),\left(T_{2}\right)$ and $\left(\mathrm{T}_{4}\right)$ than in sole whereas, the accumulated agrometeorological indices by soybean was highest in sole treatment i.e. $\left(T_{8}\right)$ than intercropped $\left(T_{3}\right)$. Significantly higher Pigeon pea equivalent yield was attained with treatment $\mathrm{T}_{3}$ followed by $\mathrm{T}_{4}$, lowest recorded in $\mathrm{T}_{1}$ intercropping system. The highest stalk / stover yield was attained by $\mathrm{T}_{2}$ as compared to sole whereas, lowest was recorded in $\mathrm{T}_{8}$. Treatment $\mathrm{T}_{3}$ performed better than other and this treatment was better in terms of growth and yield attributing characters.

\section{Introduction}

Pigoen pea is (Cajanus cajan L.) an important pulse crop, produced and consumed in India, grown during kharif season. It is a long duration crop with initial slow growth rate, so there is ample scope of growing short duration inter crops which help in getting additional yield and income. Intercropping is defined as growing two or more crops simultaneously on the same piece of land with a definite row pattern. The difficulty and risk of growing sole crops can also be minimized through intercropping. The yield is enhanced if intercropping components have different efficacies in consumption of environmental 
resources, they will make better use of the resources (Willey, 1979). Intercropping increased the amount of absorption PAR, light interception and shading in intercropping compared to sole (Ghanbari et al., 2010). Intercropping helps in variation of microclimate, especially for light intensity, relative humidity and temperature (He et al., 2012).

Light is an important competition factor since it can't be stored and is lost if not absorbed (Awal et al., 2006). Rao, (1982) reported that decrement in pods per plant in intercropping might be due to competition for light, nutrients, and moisture which reduced the growth of component crop. The adoption of cropping system that either increases the interception of radiation or maintains higher radiation use efficiency productivity per unit incident radiation might be improved. The intercrops produced substantially smaller fresh weights in either planting patter compared to the yields of sole crop. However, intercropping system as a whole resulted in higher economic returns as compared to the sole crop.

\section{Materials and Methods}

A field experiment was conducted during the kharif season of 2019-20 also the weather data for the relevant period of experiment recorded at experimental farm of the Department of Agricultural Meteorology located at College of Agriculture, Vasantrao Naik Marathwada Krishi Vidyapeeth, Parbhani. The field selected for experiment is uniform with typical black cotton soil. The daily observations converted on weekly basis and used for analysis.

In the present investigation Randomized Block Design (RBD) used with single sowing date (2 July 2019) at the nine treatments with three replication of different crops with varieties were tried. The size of each plot was $4.5 \mathrm{~m} \times 5.4 \mathrm{~m}$. The crops used in the experiment were Pigeon pea (BDN-711), Sorghum (Parbhani Shakti), Maize (Dekalb), Sesamum (MAUS-158) and Soybean (JLT408). $\mathrm{T}_{1}$ (Pigeon pea + Sorghum), $\mathrm{T}_{2}$ (Pigeon pea + Maize $), T_{3}$ (Pigeon pea + Soybean $), T_{4}$ (Pigeon pea + Sesamum), $\mathrm{T}_{5}$ (Pigeon pea), $\mathrm{T}_{6}$ (Sorghum), $\mathrm{T}_{7}$ (Maize), $\mathrm{T}_{8}$ (Soybean) and $\mathrm{T}_{9}$ (Sesamum) treatments were used with 4:2 ratio of component crop.

On the basis of Govt. minimum support price (MSP) of pigeon pea, sorghum, maize, soybean and maize seed the yield of each treatment for both component crops converted into crop equivalent yield of pigeon pea crop. The pigeon pea equivalent (PYE) $\left(\mathrm{Kg} \mathrm{ha}^{-1}\right)$ is calculated as follows:

$\mathrm{PEY}=\mathrm{P}_{\mathrm{ab}}+\stackrel{\mathrm{N}_{\mathrm{ab}} \times \mathrm{N}_{\mathrm{mp}}}{-\cdot-\cdot-\cdot-\cdot-}$

Where,

$\mathrm{PEY}=$ Pigeon pea equivalent yield $\left(\mathrm{Kg} \mathrm{ha}^{-1}\right)$, $\mathrm{P}_{\mathrm{ab}}=$ Yield of pigeon pea in intercropping system $\left(\mathrm{Kg} \mathrm{ha}{ }^{-1}\right), \mathrm{N}_{\mathrm{ab}}=$ Yield of intercrop (sorghum, maize, soybean and sesamum) in intercropping system $\left(\mathrm{Kg} \mathrm{ha}^{-1}\right), \mathrm{N}_{\mathrm{mp}}=$ Market price of intercrop (sorghum, maize, soybean and Sesamum) $\left(\mathrm{Rs} \mathrm{Kg}^{-1}\right), \mathrm{P}_{\mathrm{mp}}=$ Pigeon pea market price $\left(\mathrm{Rs} \mathrm{Kg}^{-1}\right)$

\section{Computation of Agrometeorological indices (GDD, HTU and PTU)}

\section{Growing Degree Days (GDD)}

( C day)

Growing Degree Days is defined as "the sum over the growing season of a crop of the difference between the daily temperature and a reference temperature". The total GDD for 
different phenophases were determined by the following formula. (Nuttonson, 1955). The base temperature $\left(8^{\circ} \mathrm{C}\right)$ for all the crops in the study, following Schenkler and Roberts, (2009). This is because the literature indicates that the base temperature for most crops ranges between 5 to $10^{\circ} \mathrm{C}$ (Luo, 2011).

$\mathrm{GDD}=\sum[(\mathrm{Tmax}+\mathrm{Tmin}) / 2]-\mathrm{Tb}$

Where,

Tmax $=$ Daily maximum air temperature $\left({ }^{\circ} \mathrm{C}\right)$, Tmin = Daily minimum air temperature $\left({ }^{\circ} \mathrm{C}\right)$, $\mathrm{Tb}=$ Base temperature $\left({ }^{\circ} \mathrm{C}\right)$

\section{Helio Thermal Units (HTU)}

\section{( C day hrs)}

The HTU may be defined as "the accumulated product of GDD and bright sunshine hours between the developmental thresholds for each day." The sum of HTU for each phenophase was worked out by following equation (Singh et al., 1990).

$\mathrm{HTU}=\mathrm{GDD} \times$ Mean BSS

Where,

BSS $=$ Bright Sunshine Hours

Photo Thermal Units (PTU)

\section{( C day hrs)}

PTU may be defined as "the product of growing degree days and the day length." PTU was computed by using following formula. (Gudadhe et al., 2013). Day length for sorghum, soybean, sesamum and pigeon pea (10 hrs) and for maize (12 hrs). (Qiang et al., 2015).

$\mathrm{PTU}=\mathrm{GDD} \times$ Day length

\section{Results and Discussion}

The effect of weather parameters on crop physiology was expressed directly on the phenology. The duration required for each phenophase as influenced by different treatments. In the present investigation, the whole life cycle of crops (from sowing to physiological maturity) was divided into various distinct phenophases on the basis of external morphological characteristics. Thermal time is widely used for describing the temperature responses to growth and development of crops. GDD, HTU and PTU required for completion of different phenophases of pigeon pea were worked out.

\section{Agrometeorological Indices}

\section{Accumulated GDD, HTU and PTU in Pigeon pea}

The data in table 1 revealed the highest total agrometeorological indices was accumulated by treatment $\left(T_{1}\right)$ i.e. pigeon pea intercropped with sorghum as compared to treatment $\left(\mathrm{T}_{5}\right)$ i.e. sole pigeon pea. The result indicated that the total GDD, HTU and PTU accumulated from sowing to physiological maturity ranged between 3988.3 to $4315.7^{\circ} \mathrm{C}$ day, 22907.1 to $25021.4^{\circ} \mathrm{C}$ day hrs and 39883.0 to $43156.5^{\circ} \mathrm{C}$ day hrs among the treatments $\mathrm{T}_{1}, \mathrm{~T}_{2}, \mathrm{~T}_{3}, \mathrm{~T}_{4}$ and $\mathrm{T}_{5}$ respectively.

\section{Accumulated GDD, HTU and PTU in Sorghum}

The data in table 2 revealed that the highest total agrometeorological indices was accumulated by treatment $\left(\mathrm{T}_{1}\right)$ i.e. pigeon pea intercropped with sorghum as compared to treatment $\left(\mathrm{T}_{6}\right)$ i.e. sole sorghum. The result indicated that the total GDD, HTU and PTU accumulated from sowing to physiological maturity ranged between 3006.5 to $3109.8^{\circ} \mathrm{C}$ day, 15299.8 to $16143.9^{\circ} \mathrm{C}$ day hrs and 
30065.0 to $31097.5^{\circ} \mathrm{C}$ day hrs among the treatment $\mathrm{T}_{1}$ and $\mathrm{T}_{6}$ respectively.

Accumulated GDD, HTU and PTU in Maize

The data in table 3 revealed that the the highest total agrometeorological indices was accumulated by treatment $\left(\mathrm{T}_{2}\right)$ i.e. pigeon pea intercropped with maize as compared to treatment $\left(T_{7}\right)$ i.e. sole maize. The result indicated that the total GDD, HTU and PTU accumulated from sowing to physiological maturity ranged between 2641.3 to $2743.6^{\circ} \mathrm{C}$ day, 13284.8 to $14029.6^{\circ} \mathrm{C}$ day hrs and 31695.6 to $32923.2^{\circ} \mathrm{C}$ day hrs among the treatment $\mathrm{T}_{2}$ and $\mathrm{T}_{7}$ respectively.

Table.1 Accumulated GDD, HTU and PTU in Pigeon pea

\begin{tabular}{|c|c|c|c|c|c|c|}
\hline \multicolumn{7}{|c|}{ Growing Degree Days } \\
\hline \multirow[t]{2}{*}{ Treatment } & \multicolumn{6}{|c|}{ Phenophases } \\
\hline & $\mathbf{P}_{1}$ & $\mathbf{P}_{2}$ & $\mathbf{P}_{3}$ & $\mathbf{P}_{4}$ & $\mathbf{P}_{5}$ & Total \\
\hline $\mathbf{T}_{1}:$ (Pigeonpea + Sorghum) & 247.3 & 1228.5 & 2816.4 & 3055.4 & 4315.7 & 4315.7 \\
\hline $\mathbf{T}_{2}:$ (Pigeonpea + Maize) & 247.3 & 1228.5 & 2791.8 & 3030.8 & 4274.6 & 4274.6 \\
\hline $\mathbf{T}_{3}$ : (Pigeonpea + Soybean) & 247.3 & 1201.9 & 2742.0 & 2957.0 & 4184.7 & 4184.7 \\
\hline $\mathbf{T}_{4}:$ (Pigeonpea + Sesamum) & 247.3 & 1174.9 & 2690.7 & 2882.7 & 4071.9 & 4071.9 \\
\hline$T_{5}:$ (Sole Pigeon pea) & 247.3 & 1148.5 & 2639.1 & 2808.1 & 3988.3 & 3988.3 \\
\hline Gmean & 247.3 & 1196.5 & 2736.0 & 2946.8 & 4167.0 & - \\
\hline \multicolumn{7}{|c|}{ Helio Thermal Units } \\
\hline \multirow[t]{2}{*}{ Treatment } & \multicolumn{6}{|c|}{ Phenophases } \\
\hline & $\mathbf{P}_{1}$ & $\mathbf{P}_{2}$ & $\mathbf{P}_{\mathbf{3}}$ & $\mathbf{P}_{4}$ & $\mathbf{P}_{5}$ & Total \\
\hline $\mathbf{T}_{1}:($ Pigeonpea + Sorghum) & 840.8 & 5158.1 & 14367.6 & 15443.1 & 25021.4 & 25021.4 \\
\hline $\mathbf{T}_{2}:($ Pigeonpea + Maize $)$ & 840.8 & 5158.1 & 14225.5 & 15372.7 & 24825.2 & 24825.2 \\
\hline $\mathbf{T}_{3}:$ (Pigeonpea + Soybean) & 840.8 & 4945.7 & 13724.0 & 14949.5 & 24156.9 & 24156.9 \\
\hline $\mathbf{T}_{4}:$ (Pigeonpea + Sesamum) & 840.8 & 4922.2 & 13562.0 & 14810.0 & 23491.2 & 23491.2 \\
\hline T5: (Sole Pigeon pea) & 840.8 & 4896.2 & 13243.6 & 14409.7 & 22907.1 & 22907.1 \\
\hline Gmean & 840.8 & 5016.1 & 13824.5 & 14997.0 & 24080.4 & - \\
\hline \multicolumn{7}{|c|}{ Photo Thermal Units } \\
\hline \multirow[t]{2}{*}{ Treatment } & \multicolumn{6}{|c|}{ Phenophases } \\
\hline & $\mathbf{P}_{1}$ & $\mathbf{P}_{2}$ & $\mathbf{P}_{\mathbf{3}}$ & $\mathbf{P}_{4}$ & $\mathbf{P}_{5}$ & Total \\
\hline $\mathbf{T}_{1}:($ Pigeonpea + Sorghum $)$ & 2473.0 & 12285.0 & 28163.5 & 30553.5 & 43156.5 & 43156.5 \\
\hline $\mathbf{T}_{2}:$ (Pigeonpea + Maize) & 2473.0 & 12285.0 & 27918.4 & 30308.4 & 42745.9 & 42745.9 \\
\hline $\mathbf{T}_{\mathbf{3}}$ : (Pigeonpea + Soybean) & 2473.0 & 12019.0 & 27419.5 & 29569.5 & 41846.0 & 41846.0 \\
\hline $\mathbf{T}_{4}:($ Pigeonpea + Sesamum) & 2473.0 & 11749.0 & 26906.5 & 28826.5 & 40718.5 & 40718.5 \\
\hline $\mathrm{T}_{5}:$ (Sole Pigeon pea) & 2473.0 & 11485.0 & 26391.0 & 28081.0 & 39883.0 & 39883.0 \\
\hline Gmean & 2473.0 & 11964.6 & 27359.8 & 29467.8 & 41670.0 & - \\
\hline
\end{tabular}

$\mathrm{P}_{1}$ - Sowing to emergence $\mathrm{P}_{2}-$ Emergence to branching

$\mathrm{P}_{3}$ - Branching to flowering $\mathrm{P}_{4}$ - Flowering to pod formation

$\mathrm{P}_{5}-$ Pod formation to physiological maturity 
Table.2 Accumulated GDD, HTU and PTU in Sorghum

\begin{tabular}{|c|c|c|c|c|c|c|c|c|}
\hline \multicolumn{9}{|c|}{ Growing Degree Days } \\
\hline \multirow[t]{2}{*}{ Treatment } & \multicolumn{8}{|c|}{ Phenophases } \\
\hline & $\mathbf{P}_{1}$ & $\mathbf{P}_{2}$ & $\mathbf{P}_{3}$ & $\mathbf{P}_{4}$ & $\mathbf{P}_{5}$ & $\mathbf{P}_{6}$ & $\mathbf{P}_{7}$ & Total \\
\hline T1:(Pigoenpea+Sorghum) & 160.5 & 1395.2 & 1703.7 & 1978.3 & 2208.4 & 2462.0 & 3109.8 & 3109.8 \\
\hline T6:(Sole Sorghum) & 160.5 & 1392.0 & 1676.9 & 1950.5 & 2150.7 & 2381.2 & 3006.5 & 3006.5 \\
\hline G mean & 160.5 & 1393.6 & 1690.3 & 1964.4 & 2179.6 & 2421.6 & 3058.2 & - \\
\hline \multicolumn{9}{|c|}{ Helio Thermal Units } \\
\hline \multirow[t]{2}{*}{ Treatment } & \multicolumn{8}{|c|}{ Phenophases } \\
\hline & $\mathbf{P}_{1}$ & $\mathbf{P}_{2}$ & $\mathbf{P}_{\mathbf{3}}$ & $\mathbf{P}_{4}$ & $\mathbf{P}_{5}$ & $\mathbf{P}_{6}$ & $\mathbf{P}_{7}$ & Total \\
\hline T1:(Pigoenpea+Sorghum) & 497.6 & 6547.4 & 8213.3 & 9064.6 & 10510.1 & 12386.7 & 16143.9 & 16143.9 \\
\hline T6:(Sole Sorghum) & 497.6 & 6285.7 & 8052.1 & 8818.2 & 9832.4 & 11422.9 & 15299.8 & 15299.8 \\
\hline G mean & 497.6 & 6416.6 & 8132.7 & 8941.4 & 10171.3 & 11904.8 & 15721.9 & - \\
\hline \multicolumn{9}{|c|}{ Photo Thermal Units } \\
\hline \multirow[t]{2}{*}{ Treatment } & \multicolumn{8}{|c|}{ Phenophases } \\
\hline & $\mathbf{P}_{1}$ & $\mathbf{P}_{2}$ & $\mathbf{P}_{\mathbf{3}}$ & $\mathbf{P}_{4}$ & $\mathbf{P}_{5}$ & $\mathbf{P}_{6}$ & $\mathbf{P}_{7}$ & Total \\
\hline T1:(Pigoenpea+Sorghum) & 1605.0 & 13951.5 & 17036.5 & 19782.5 & 22083.5 & 24619.5 & 31097.5 & 31097.5 \\
\hline T6:(Sole Sorghum) & 1605.0 & 13920.0 & 16769.0 & 19505.0 & 21507.0 & 23812.0 & 30065.0 & 30065.0 \\
\hline G mean & 1605.0 & 13935.8 & 16902.8 & 19643.8 & 21795.3 & 24125.8 & 30581.3 & - \\
\hline
\end{tabular}

Table.3 Accumulate GDD, HTU and PTU in Maize

\begin{tabular}{|c|c|c|c|c|c|c|c|}
\hline \multicolumn{8}{|c|}{ Growing Degree Days } \\
\hline \multirow[t]{2}{*}{ Treatment } & \multicolumn{7}{|c|}{ Phenophases } \\
\hline & $\mathbf{P}_{1}$ & $\mathbf{P}_{2}$ & $\mathbf{P}_{3}$ & $\mathbf{P}_{4}$ & $\mathbf{P}_{5}$ & $\mathbf{P}_{6}$ & Total \\
\hline $\mathrm{T}_{2}:($ Pigeonpea+Maize $)$ & 385.7 & 1314.5 & 1522.8 & 1644.7 & 2365.0 & 2743.6 & 2743.6 \\
\hline $\mathbf{T}_{7}:($ Sole Maize $)$ & 385.7 & 1283.0 & 1465.2 & 1567.0 & 2285.2 & 2641.3 & 2641.3 \\
\hline G mean & 385.7 & 1298.8 & 1494.0 & 1605.9 & 2325.1 & 2692.5 & - \\
\hline \multicolumn{8}{|c|}{ Helio Thermal Units } \\
\hline \multirow[t]{2}{*}{ Treatment } & \multicolumn{7}{|c|}{ Phenophases } \\
\hline & $\mathbf{P}_{1}$ & $\mathbf{P}_{2}$ & $\mathbf{P}_{3}$ & $\mathbf{P}_{4}$ & $\mathbf{P}_{5}$ & $\mathbf{P}_{6}$ & Total \\
\hline $\mathbf{T}_{2}:($ Pigeonpea+Maize $)$ & 1812.8 & 6085.3 & 7418.4 & 8027.9 & 11341.3 & 14029.6 & 14029.6 \\
\hline $\mathbf{T}_{7}:($ Sole Maize $)$ & 1812.8 & 5671.2 & 6946.6 & 7669.8 & 10614.0 & 13284.8 & 13284.8 \\
\hline G mean & 1812.8 & 5878.3 & 7182.5 & 7848.9 & 10977.7 & 13657.2 & - \\
\hline \multicolumn{8}{|c|}{ Photo Thermal Units } \\
\hline \multirow[t]{2}{*}{ Treatment } & \multicolumn{7}{|c|}{ Phenophases } \\
\hline & $\mathbf{P}_{1}$ & $\mathbf{P}_{2}$ & $\mathbf{P}_{3}$ & $\mathbf{P}_{4}$ & $\mathbf{P}_{5}$ & $\mathbf{P}_{6}$ & Total \\
\hline $\mathrm{T}_{2}:($ Pigeonpea+Maize $)$ & 4628.4 & 15774.0 & 18273.6 & 19736.4 & 28380.0 & 32923.2 & 32923.2 \\
\hline $\mathbf{T}_{7}:($ Sole Maize $)$ & 4628.4 & 15396.0 & 17582.4 & 18804.0 & 27422.4 & 31695.6 & 31695.6 \\
\hline G mean & 4628.4 & 15585.0 & 17928.0 & 19270.2 & 27901.2 & 32309.4 & - \\
\hline $\begin{array}{l}\mathrm{P}_{1}-\text { Sowing to seedling } \\
\mathrm{P}_{3}-\text { Grandgrowth to tasseling } \\
\mathrm{P}_{5}-\text { Silking to milk stage }\end{array}$ & $\begin{array}{l}\mathrm{P}_{2}-\text { Seed } \\
\mathrm{P}_{4}-\text { Tasse } \\
\mathrm{P}_{6}-\text { Milk }\end{array}$ & $\begin{array}{l}\text { ig to grandg } \\
\text { ng to silkin } \\
\text { tage to phys }\end{array}$ & 1 maturity & & & & \\
\hline
\end{tabular}


Table.4 Agrometeorological Indices (GDD, HTU and PTU) in Soybean

\begin{tabular}{|c|c|c|c|c|c|c|c|}
\hline \multicolumn{8}{|c|}{ Growing Degree Days } \\
\hline \multirow[t]{2}{*}{ Treatment } & \multicolumn{7}{|c|}{ Phenophases } \\
\hline & $\mathbf{P}_{1}$ & $\mathbf{P}_{2}$ & $\mathbf{P}_{\mathbf{3}}$ & $\mathbf{P}_{4}$ & $\mathbf{P}_{5}$ & $\mathbf{P}_{6}$ & Total \\
\hline$T_{3}:($ Pigeonpea+Soybean) & 644.1 & 1446.7 & 1628.2 & 1773.2 & 1942.6 & 2537.3 & 2537.3 \\
\hline $\mathbf{T}_{8}:($ Sole Soybean) & 644.1 & 1503.6 & 1705.6 & 1878.6 & 2100.7 & 2721.3 & 2721.3 \\
\hline G mean & 644.1 & 1475.2 & 1666.9 & 1825.9 & 2021.7 & 2629.3 & - \\
\hline \multicolumn{8}{|c|}{ Helio Thermal Units } \\
\hline \multirow[t]{2}{*}{ Treatment } & \multicolumn{7}{|c|}{ Phenophases } \\
\hline & $\mathbf{P}_{1}$ & $\mathbf{P}_{2}$ & $\mathbf{P}_{\mathbf{3}}$ & $\mathbf{P}_{4}$ & $\mathbf{P}_{5}$ & $\mathbf{P}_{6}$ & Total \\
\hline$T_{3}:($ Pigeonpea+Soybean) & 3864.6 & 6914.5 & 8239.5 & 8543.9 & 9018.2 & 12764.8 & 12764.8 \\
\hline$T_{8}:($ Sole Soybean) & 3864.6 & 7302.6 & 8393.4 & 8739.4 & 9694.4 & 13914.5 & 13914.5 \\
\hline G mean & 3864.6 & 7108.6 & 8316.5 & 8641.7 & 9356.3 & 13339.7 & - \\
\hline \multicolumn{8}{|c|}{ Photo Thermal Units } \\
\hline \multirow[t]{2}{*}{ Treatment } & \multicolumn{7}{|c|}{ Phenophases } \\
\hline & $\mathbf{P}_{1}$ & $\mathbf{P}_{2}$ & $\mathbf{P}_{3}$ & $\mathbf{P}_{4}$ & $\mathbf{P}_{5}$ & $\mathbf{P}_{6}$ & Total \\
\hline $\mathbf{T}_{3}$ :(Pigeonpea+Soybean) & 6441.0 & 14467.0 & 16282.0 & 17732.1 & 19425.9 & 25373.2 & 25373.2 \\
\hline$T_{8}:($ Sole Soybean) & 6441.0 & 15035.9 & 17055.8 & 18785.7 & 21007.0 & 27213.0 & 27213.0 \\
\hline G mean & 6441.0 & 14751.5 & 16668.9 & 18258.9 & 20216.5 & 26293.1 & - \\
\hline $\begin{array}{l}\mathrm{P}_{1}-\text { Sowing to seedling } \\
\mathrm{P}_{3}-\text { Branching to flowering } \\
\mathrm{P}_{5}-\text { Pod formation to grain format }\end{array}$ & \multicolumn{7}{|c|}{$\begin{array}{l}\mathrm{P}_{2}-\text { Seedling to branching } \\
\mathrm{P}_{4}-\text { Flowering to pod formation } \\
\mathrm{P}_{6}-\text { Grain formation to physical }\end{array}$} \\
\hline
\end{tabular}

Table.5 Accumulated GDD, HTU and PTU in Sesamum

\begin{tabular}{|c|c|c|c|c|c|c|}
\hline \multicolumn{7}{|c|}{ Growing Degree Days } \\
\hline \multirow[t]{2}{*}{ Treatment } & \multicolumn{6}{|c|}{ Phenophases } \\
\hline & $\mathbf{P}_{1}$ & $\mathbf{P}_{2}$ & $\mathbf{P}_{3}$ & $\mathbf{P}_{4}$ & $\mathbf{P}_{5}$ & Total \\
\hline $\mathbf{T}_{4}:$ (Pigeonpea+Sesamum) & 134.3 & 664.7 & 883.0 & 1163.3 & 2447.3 & 2447.3 \\
\hline T: & 134.3 & 636.2 & 830.7 & 1082.0 & 2339.1 & 2339.1 \\
\hline G mean & 134.3 & 650.5 & 856.9 & 1122.7 & 2393.2 & - \\
\hline \multicolumn{7}{|c|}{ Helio Thermal Units } \\
\hline \multirow{2}{*}{ Treatment } & \multicolumn{6}{|c|}{ Phenophases } \\
\hline & $\mathbf{P}_{1}$ & $\mathbf{P}_{2}$ & $\mathbf{P}_{3}$ & $\mathbf{P}_{4}$ & $\mathbf{P}_{5}$ & Total \\
\hline $\mathbf{T}_{4}$ :(Pigeonpea+Sesamum) & 456.6 & 4063.3 & 4390.8 & 5203.7 & 12131.6 & 12131.6 \\
\hline T9:Sole Sesamum) & 456.6 & 3819.3 & 4150.0 & 4903.9 & 11189.2 & 11189.2 \\
\hline G mean & 456.6 & 3941.3 & 4270.4 & 5053.8 & 11660.4 & - \\
\hline \multicolumn{7}{|c|}{ Photo Thermal Units } \\
\hline \multirow[t]{2}{*}{ Treatment } & \multicolumn{6}{|c|}{ Phenophases } \\
\hline & $\mathbf{P}_{1}$ & $\mathbf{P}_{2}$ & $\mathbf{P}_{3}$ & $\mathbf{P}_{4}$ & $\mathbf{P}_{5}$ & Total \\
\hline$T_{4}:($ Pigeonpea+Sesamum) & 1343.1 & 6647.2 & 8830.0 & 11633.2 & 24472.7 & 24472.7 \\
\hline T9:Sole Sesamum) & 1343.1 & 6361.6 & 8306.9 & 10820.1 & 23391.0 & 23391.0 \\
\hline G mean & 1343.1 & 6504.4 & 8568.5 & 11226.7 & 23931.9 & - \\
\hline $\begin{array}{l}P_{1}-\text { Sowing to emergence } \\
P_{3}-\text { Branching to flowering } \\
P_{5}-\text { Capsules to physical maturity }\end{array}$ & $\begin{array}{l}2 \text {-Emerger } \\
4 \text {-Flowerir }\end{array}$ & $\begin{array}{l}\text { to branchin } \\
\text { o capsules }\end{array}$ & & & & \\
\hline
\end{tabular}


Table.6 Seed yield of crops

\begin{tabular}{|c|c|c|c|}
\hline \multirow{2}{*}{ Treatments } & \multicolumn{3}{|c|}{ Seed yield $\left(\mathrm{Kg} \mathrm{h}^{\mathbf{- 1}}\right)$} \\
\cline { 2 - 4 } & Main & Inter & Pig. Equi. Yield \\
\hline $\mathbf{T}_{\mathbf{1}}$ (Pigeonpea + Sorghum) & 597 & 602 & 861 \\
\hline $\mathbf{T}_{\mathbf{2}}$ (Pigeonpea + Maize) & 837 & 1453 & 1277 \\
\hline $\mathbf{T}_{\mathbf{3}}$ (Pigeonpea + Soybean) & 1283 & 345 & 1503 \\
\hline $\mathbf{T}_{\mathbf{4}}$ (Pigeonpea + Sesamum) & 1292 & 99 & 1402 \\
\hline $\mathbf{T}_{\mathbf{5}}$ (Sole Pigeon pea) & 1349 & - & 1249 \\
\hline $\mathbf{T}_{\mathbf{6}}$ (Sole Sorghum) & 3280 & - & 3280 \\
\hline $\mathbf{T}_{\mathbf{7}}$ (Sole Maize) & 4108 & - & 4108 \\
\hline $\mathbf{T}_{\mathbf{8}}$ (Sole Soybean) & 1410 & - & 1410 \\
\hline $\mathbf{T}_{\mathbf{9}}$ (Sole Sesamum) & 285 & - & 285 \\
\hline S.E \pm & - & - & 54.62 \\
\hline CD at 5\% & - & - & 165.16 \\
\hline G Mean & 1604.6 & 624.8 & 1260.8 \\
\hline
\end{tabular}

Table.7 Stalk/Stover yield of crops

\begin{tabular}{|c|c|c|c|}
\hline \multirow{2}{*}{ Treatments } & \multicolumn{3}{|c|}{ Stalk/Stover yield(Kg ha ${ }^{-1}$ ) } \\
\cline { 2 - 4 } & Main & Inter & Pig. Equi. Yield \\
\hline $\mathbf{T}_{\mathbf{1}}$ (Pigeonpea + Sorghum) & 1970 & 589 & 2559 \\
\hline $\mathbf{T}_{\mathbf{2}}$ (Pigeonpea + Maize) & 1960 & 1128 & 3088 \\
\hline $\mathbf{T}_{\mathbf{3}}$ (Pigeonpea + Soybean) & 2080 & 640 & 2720 \\
\hline $\mathbf{T}_{\mathbf{4}}$ (Pigeonpea + Sesamum) & 2096 & 162 & 2258 \\
\hline $\mathbf{T}_{\mathbf{5}}$ (Sole Pigeon pea) & 2120 & - & 2120 \\
\hline $\mathbf{T}_{\mathbf{6}}$ (Sole Sorghum) & 2284 & - & 2284 \\
\hline $\mathbf{T}_{\mathbf{7}}$ (Sole Maize) & 2884 & - & 2884 \\
\hline $\mathbf{T}_{\mathbf{8}}$ (Sole Soybean) & 1418 & - & 1418 \\
\hline $\mathbf{T}_{\mathbf{9}}$ (Sole Sesamum) & 1734 & - & 1734 \\
\hline G Mean & 2060.7 & 629.8 & 2340.6 \\
\hline
\end{tabular}

Accumulated GDD, HTU and PTU in Soybean

The data in table 4 revealed the highest total agrometeorological indices was accumulated by treatment $\left(\mathrm{T}_{8}\right)$ i.e. sole soybean as compared to treatment $\left(\mathrm{T}_{3}\right)$ i.e. pigeon pea intercropped with soybean. The result indicated that the total GDD, HTU and PTU accumulated from sowing to physiological maturity ranged between 2537.3 to $2721.3^{\circ} \mathrm{C}$ day, 12764.8 to $13914.5^{\circ} \mathrm{C}$ day $\mathrm{hrs}$ and
25373.2 to $27213.0^{\circ} \mathrm{C}$ day hrs among the treatment $\left(\mathrm{T}_{3}\right.$ to $\left.\mathrm{T}_{8}\right)$.

Accumulated GDD, HTU and PTU in Sesamum

The data in table 5 revealed that the highest total agrometeorological indices was accumulated by treatment $\left(T_{4}\right)$ i.e. pigeon pea intercropped with sesamum as compared to treatment $\left(T_{9}\right)$ i.e. sole sesamum. The result indicated that the total GDD, HTU and PTU 
accumulated from sowing to physiological maturity ranged between 2339.1 to $2447.3^{\circ} \mathrm{C}$ day, 11189.2 to $12131.6^{\circ} \mathrm{C}$ day hrs and 23391.0 to $24472.7^{\circ} \mathrm{C}$ day hrs among the treatment $\left(\mathrm{T}_{4}\right.$ to $\left.\mathrm{T}_{9}\right)$.

\section{Yield Parameter}

\section{Seed/Grain yield $\left(\mathrm{Kg} \mathrm{ha}^{-1}\right)$}

The data from Table 6 shows that mean pigeon pea equivalent yield (PEY) $\left(\mathrm{Kg} \mathrm{ha}^{-1}\right)$ was significantly influenced by different treatment. The pigeon pea yield was significantly affected by the intercropping pattern. There was a significant difference between PEY with various pigeon pea based intercropping systems. The PEY was obtained highest from the pigeon pea + soybean $\left(\mathrm{T}_{3}\right)$ intercropping system $\left(1503 \mathrm{Kg} \mathrm{ha}^{-1}\right)$, followed by the pigeon pea + sesamum $\left(\mathrm{T}_{4}\right)$ intercropping system (1402 $\left.\mathrm{Kg} \mathrm{ha}^{-1}\right)$, pigeon pea + maize $\left(\mathrm{T}_{2}\right)$ intercropping system (1277) than the sole pigeon pea $\left(\mathrm{T}_{5}\right)\left(1249 \mathrm{Kg} \mathrm{ha}^{-1}\right)$. Whereas, the PEY was obtained lowest from pigeon pea + sorghum $\left(\mathrm{T}_{1}\right)$ intercropping system (861 Kg ha ${ }^{-1}$ ) as compared to sole pigeon pea. However, in the PEY it was observed that the treatment $\mathrm{T}_{3}$ was significantly superior than other treatment, which was at par with the treatment $\mathrm{T}_{4}$.

The legume and legume as main crop and intercrop might have symbiotic effect with each other and reduced the competition for moisture and nutrients between the component crops and significantly increased yield of both component crops. Hence, the PEY from pigeon pea + soybean intercropping treatment was highest. Similar result was reported by Waghmare et al., (1982) and Kathmale et al., (2014). Sole pigeon pea seemed to have been more remunerative than pigeon pea + sorghum intercropping probably because of intense inter - specific competition from the sorghum component and subsequently reduced profits.
On the other hand, maize crop adversely affected the yield of pigeon pea however the PEY was more than sole pigeon pea because of good yield of maize but it could not compensate the losses occurred due to reduced pigeon pea yield. Similar result was observed by Egbe and Idoko, (2012) and Tiwari et al., (2012). So it was observed that sole pigeon pea gave significantly higher grain yield than the intercropped. However, intercropping systems gave higher PEY over sole pigeon pea except from pigeonpea + sorghum intercropping treatment. Similar results were observed by Ito et al., (1993), Singh and Singh, (1994), Rekha and Dhurua, (2009), and Reddy et al., (2015).

\section{Stalk/Stover yield $\left(\mathrm{Kg} \mathrm{ha}^{-1}\right)$}

The data from Table 7 shows that mean pigeonpea stalk/stover yield $\left(\mathrm{Kg} \mathrm{ha}{ }^{-1}\right)$ was significantly influenced by different treatments. The stalk/stover yield was significantly affected by the intercropping patter. It was significantly superior in treatment $\mathrm{T}_{2}\left(3088 \mathrm{Kg} \mathrm{ha}^{-1}\right)$ i.e. pigoenpea + maize as compared to all treatment and lowest in treatment $\mathrm{T}_{8}\left(1418 \mathrm{Kg} \mathrm{ha}^{-1}\right)$ i.e. sole soybean. Among pigeon pea based intercropping systems, higher stover yield of sorghum as an intercrop was recorded in pigeon pea + sorghum system. Hence, the pigeon pea stalk/stover yield under Pigeon Pea + sorghum was highest as compared to pigeon pea yields with other intercrops. Similar result was reported by Kathmale et al., (2014).

The highest agrometeorological indices was accumulated by treatment $\left(\mathrm{T}_{1}\right)$ i.e. pigeon pea intercropped with sorghum as compared to sole pigeon pea i.e. $\mathrm{T}_{5}$, by sorghum, maize and sesamum were highest in intercropped treatment i.e. $\left(\mathrm{T}_{1}\right),\left(\mathrm{T}_{2}\right)$ and $\left(\mathrm{T}_{4}\right)$ than in sole whereas, accumulated by soybean was highest in sole treatment i.e. $\left(\mathrm{T}_{8}\right)$ than intercropped $\left(\mathrm{T}_{3}\right)$. Variations in agrometeorological indices 
for various phonological stages indicated that accumulated temperature can be utilized for dry biomass and crop yield forecast. Significantly higher PEY was attained with treatment $\mathrm{T}_{3}$ (Pigeon pea + Soybean) followed by $\mathrm{T}_{4}$ (Pigeon pea + Sesamum). This might be due to no competition between main crop and intercrop for growth, development and for above ground and below ground resources as crop was of shorter duration. The lowest PEY was recorded in $\left(T_{1}\right)$ pigeon pea + sorghum intercropping system and might be due to intense inter-specific competition. In the pigeon pea based intercropping system, highest stalk/stover yield were attained by $T_{2}$ i.e. pigeon pea intercropped with maize as compared to sole whereas, lowest stalk/stover yield was recorded in $\mathrm{T}_{8}$ i.e. sole soybean. Treatment $T_{3}$ performed better than other and this treatment was better in terms of growth and yield attributing characters.

\section{References}

Awal, M. A., Koshi, H. and Ikeda, T. (2006). Radiation interception and use by maize/peanut intercrop canopy Agr. Forest Meteorol. 139, 74-83.

Egbe, O. M. and Idoko, A. J. (2012). Evaluation of pigeonpea genotypes for intercropping with maize and sorghum in Southern Guinea Savanna: Economic benefits. International Journal of Agriculture and Forestry. 2(1): 108-114.

Ghanbari G., Dahmardeh, M., Siahsar, B. A. and Ramroudi, M. (2010).Effect of Maize (Zea mays L.)-cowpea (Vigna unguiculata L.) Intercropping on Light Distribution, Soil Temperature and Soil Moisture in Arid Environment. Journal of Food Agriculture and Environment. 8(1):102-108.

Gudadhe, N. N., Neeraj Kumar, Pisal, R. R., Mote, B. M. and Dhode, M. B.(2013).Evaluation of agro meteorological indices in relation to crop phenology of cotton (Gossippium spp.) and chickpea (Cicer aritinum L.) at Rahuri region of Maharashtra. Trends in Biosciences 6(3): 246-250.

He, H., Yang L., Liming F., Lihua, Z., Han W., Yang J., and Chengyun L. (2012). The effect of intercropping of maize and soybean on microclimate, CCTA IFIP AICT (369):257-263.

Ito, O., Matsunaga, R., Tovita, S., Rao, T. P. and Gayatri Devi, Y. (1993).Spatial distribution of root activity and nitrogen fixation in sorghum/pigeonpea intercropping on an Indian Alfisols. Plant and Soil. (155):341-344.

Kathmale, D. K., Dhadge, S. M., Stpute, S. V., Patil, G., Ravindra, C., Rao, C. S., Jadhav, J. D., and kadam, J. R. (2014). Evaluation pigeonpea based intercropping systems under Semi-arid Vertisol in Scarcity Zone of Maharashtra. Indian J. Dryland Agric. Res. and Dev. 29(1): 27-34.

Luo, Q. (2011), “Temperature Threshold and crop production: A Review," Climate Change. (109):583-98.

Nuttonson, M. Y. (1955). Wheat climatic relationship and use of phenology in ascertaining the thermal and photothermal requirements of wheat. American Institute of Crop Ecology. Washington D.C. (7), 388.

Qiang, C., Hao, Z. Xian, W. and You - Zhi, L. (2015). An insight into the sensitivity of maize to photoperiod changes under controlled conditions. Plant, Cell and Environment. (38): 1479-1489.

Rao, A. R. (1982). Intercropping in cotton. Cotton Development, 12(1/2), 33-34.

Reddy, V., Koppalkar, B. G. and Rjun, M. (2015). Growth and yield advantages of pigeonpea with sesame intercropping system influenced by nutrient management. International 
Quarterly Journal of Environmental Sciences. (7):0974-0376.

Rekha, M. S. and Dhurua, S. (2009). Productivity of pigeon pea + soybean intercropping systems as influenced by planting patterns and duration of pigeonpea varieties under rainfed conditions. Legume Res. 32(1): 51-54, 2009.

Schlenker, W., and Roberts, M. J. (2009), "Nonlinear Temperature Effects Indicate Severe Damages to U. S. Crop Yields under Climate Change," Proceedings of the National Academy of Sciences. (106): 15594-98.

Singh, G., Narwal, S. S., Rao, V. U. M. and Daiya, D. S. (1990). Effect of sowing date on requirement of growing degree days, heliothermal units and photothermal units on phenology of winter maize (Zea mays). Indian J. Agric. Sci. 60(11):723-731.

Singh, R. A. and Singh, A. K. (1994). Comparative performance of different intercropping systems with pigeonpea under rainfed conditions of Vindhya region. Indian Journal of Agronomy. (39):623-615.

Tiwari, D., Sharma, B. B. and Debey, P. K. (2012). Integrated nutrient management in pigeonpea based intercropping system under Tarai conditions of Uttarakhand. Research journal of Agricultural Sciences. 3(1); 014-018.

Waghmare, A. B., Krishan, T. K. and Singh, S. P. (1982). Crop compatibility and spatial arrangement in sorghum-based intercropping system. Journal of Agricultural Science. (Camb), (99): 621-629.

Willey, R. W. (1979). Intercropping-its importance and research needs. Part 1. Compettion and yield advantages. Field Crops Abstr. 32: 1-10.Yildirim, E. and Guvenc, I. (2005). Intercropping based on cauliflower: more productive, profitable and Highly sustainable. European Journal of Agronomy. (22):11-18.

\section{How to cite this article:}

Chatte, S. N., M. G. Jadhav, D. S. Dhekane, I. A. B. Mirza, K. K. Dakhore and More, S. S. 2021. Agrometeorological Indices, Crop Phenology and Yield of Pigeon pea as Influenced by Pigeon pea (Cajanus cajan L.) based Intercropping System. Int.J.Curr.Microbiol.App.Sci. 10(11): 387-396. doi: https://doi.org/10.20546/ijcmas.2021.1011.044 